T. Arakawa and M. Kaneko

Nagoya Math. J.

Vol. 153 (1999), 189-209

\title{
MULTIPLE ZETA VALUES, POLY-BERNOULLI NUMBERS, AND RELATED ZETA FUNCTIONS
}

\section{TSUNEO ARAKAWA AND MASANOBU KANEKO}

\begin{abstract}
We study the function

$$
\zeta\left(k_{1}, \ldots, k_{n-1} ; s\right)=\sum_{0<m_{1}<m_{2}<\cdots<m_{n}} \frac{1}{m_{1}^{k_{1}} \cdots m_{n-1}^{k_{n-1} m_{n}^{s}}}
$$

and show that the poly-Bernoulli numbers introduced in our previous paper are expressed as special values at negative arguments of certain combinations of these functions. As a consequence of our study, we obtain a series of relations among multiple zeta values.
\end{abstract}

\section{$\S 1$. Introduction}

In recent years, many authors have studied the "multiple zeta values" (or Euler-Zagier sums)

$$
\zeta\left(k_{1}, k_{2}, \ldots, k_{n}\right)=\sum_{0<m_{1}<m_{2}<\cdots<m_{n}} \frac{1}{m_{1}^{k_{1}} m_{2}^{k_{2}} \cdots m_{n}^{k_{n}}},
$$

where $k_{i}$ are positive integers and $k_{n} \geq 2$, not only in the context of number theory but also of knot theory, the theory of mixed Tate motives, and quantum field theory (see e.g. [A], [?], [BBG], [Br], [H], [LM], [Z], [Z2] and references therein). It seems to be apparent that these numbers are very interesting and important from various aspects. Don Zagier, in his work [Z] (in preparation) devoted to an extensive study of the multiple zeta values, mentions briefly the "multiple zeta functions" as well;

$$
\zeta\left(s_{1}, s_{2}, \ldots, s_{n}\right)=\sum_{0<m_{1}<m_{2}<\cdots<m_{n}} \frac{1}{m_{1}^{s_{1}} m_{2}^{s_{2}} \cdots m_{n}^{s_{n}}} \quad\left(s_{i} \in \mathbf{C}\right)
$$

in particular their analytic continuations to meromorphic functions in $\mathbf{C}^{n}$. However, no further study of $\zeta\left(s_{1}, s_{2}, \ldots, s_{n}\right)$ seems to have been made,

Received October 13, 1997. 
probably because "nice properties like the functional equation of the Riemann zeta function do not seem to extend" to $\zeta\left(s_{1}, s_{2}, \ldots, s_{n}\right)$ for $n \geq 2$.

In this paper, we study the single variable function

$$
\zeta\left(k_{1}, \ldots, k_{n-1} ; s\right)=\sum_{0<m_{1}<m_{2}<\cdots<m_{n}} \frac{1}{m_{1}^{k_{1}} \cdots m_{n-1}^{k_{n-1} m_{n}^{s}}},
$$

for the purpose of establishing a connection between the multiple zeta values and the "poly-Bernoulli numbers" introduced in [Ka]. In seeking an answer to the natural question whether the poly-Bernoulli numbers appear as special values of zeta functions of any sort, we were led into the consideration of the functions $\zeta\left(k_{1}, k_{2}, \ldots, k_{n-1} ; s\right)$. We show that the poly-Bernoulli numbers are indeed expressed as special values at negative arguments of certain combinations of the zeta functions $\zeta\left(k_{1}, k_{2}, \ldots, k_{n-1} ; s\right)$ (Theorem 6 and Corollary 7 in $\S 3$ ). As a consequence of our study, we also obtain a series of relations among multiple zeta values, namely;

Corollary of Theorem 9. For integers $m, r \geq 1$ and $k \geq 2$, we have

$$
\begin{aligned}
& \sum_{\substack{a_{1}+\ldots+a_{k}=m \\
\forall a_{j} \geq 0}}\left(\begin{array}{c}
a_{k}+r \\
r
\end{array}\right) \zeta\left(a_{1}+1, \ldots, a_{k-1}+1 ; a_{k}+r+1\right) \\
&+(-1)^{k} \sum_{\substack{a_{1}+\cdots+a_{k}=r \\
\forall a_{j} \geq 0}}\left(\begin{array}{c}
a_{k}+m \\
m
\end{array}\right) \zeta\left(a_{1}+1, \ldots, a_{k-1}+1 ; a_{k}+m+1\right) \\
&=\sum_{j=0}^{k-2}(-1)^{j} \zeta(\underbrace{1, \ldots, 1}_{r-1}, k-j) \zeta(\underbrace{1, \ldots, 1}_{m-1}, 2+j) .
\end{aligned}
$$

One recalls that, to understand a complete set of relations is one of the fundamental problems in the theory of multiple zeta values.

After some preliminary discussions on $\zeta\left(k_{1}, k_{2}, \ldots, k_{n-1} ; s\right)$ in $\S 2$, we state and prove our main result in $\S 3 . ~ \S 4$ is devoted to a generalization of the zeta function introduced in $\S 3$, and that leads to some formulas for multiple zeta values. In the final $\S 5$ we discuss some problems.

\section{$\S 2$. Single variable multiple zeta functions}

As mentioned in the introduction, we study the following zeta function 


$$
\begin{array}{r}
\zeta\left(k_{1}, k_{2}, \ldots, k_{n-1} ; s\right):=\sum_{0<m_{1}<m_{2}<\cdots<m_{n-1}<m_{n}} \frac{1}{m_{1}^{k_{1}} m_{2}^{k_{2}} \cdots m_{n-1}^{k_{n-1}} m_{n}^{s}} \\
\left(n \geq 1, k_{i} \geq 1\right)
\end{array}
$$

which is absolutely convergent for $\operatorname{Re}(s)>1$. When $n=1$, the function $\zeta\left(k_{1}, k_{2}, \ldots, k_{n-1} ; s\right)$ is understood to be the Riemann zeta function $\zeta(s)$. We mention that the function $\zeta\left(k_{1}, k_{2}, \ldots, k_{n-1} ; s\right)$ is, as a Dirichlet series, a generating series of "multiple harmonic sums" (finite truncation of multiple zeta values) recently studied by M. Hoffman and C. Moen:

$$
\zeta\left(k_{1}, k_{2}, \ldots, k_{n-1} ; s\right)=\sum_{m=n}^{\infty} \frac{A_{m}\left(k_{1}, k_{2}, \ldots, k_{n-1}\right)}{m^{s}}
$$

where

$$
A_{m}\left(k_{1}, k_{2}, \ldots, k_{n-1}\right)=\sum_{0<m_{1}<m_{2}<\cdots<m_{n-1}<m} \frac{1}{m_{1}^{k_{1}} m_{2}^{k_{2}} \cdots m_{n-1}^{k_{n-1}}} .
$$

We shall give an integral representation and the analytic continuation of $\zeta\left(k_{1}, k_{2}, \ldots, k_{n-1} ; s\right)$, both of which are carried out by a standard method. Before proceeding to the statement, we introduce the "multi-logarithmic function" defined by

$$
L i_{k_{1}, k_{2}, \ldots, k_{n}}(z):=\sum_{0<m_{1}<m_{2}<\cdots<m_{n}} \frac{z^{m_{n}}}{m_{1}^{k_{1}} m_{2}^{k_{2}} \cdots m_{n}^{k_{n}}} \quad\left(k_{i} \geq 1,|z|<1\right),
$$

and give a lemma and a proposition which will be needed in the proof of the theorem and also in the later discussion.

\section{LEMMA 1. (i)}

$$
\frac{d}{d z} L i_{k_{1}, k_{2}, \ldots, k_{n}}(z)= \begin{cases}\frac{1}{z} L i_{k_{1}, k_{2}, \ldots, k_{n-1}, k_{n}-1}(z), & \text { for } k_{n}>1 \\ \frac{1}{1-z} L i_{k_{1}, k_{2}, \ldots, k_{n-1}}(z), & \text { for } k_{n}=1\end{cases}
$$

(ii) $L \underbrace{i_{1,1, \ldots, 1}}_{n}(z)=\frac{1}{n !}(-\log (1-z))^{n}$. 
Proof. (i) is straightforward, and (ii) is derived from (i) and $L i_{1}(z)=$ $-\log (1-z)$ by integrating repeatedly.

Proposition 2. (i) For $\operatorname{Re}(s)>1$, we have

$$
\zeta\left(k_{1}, k_{2}, \ldots, k_{n-1} ; s\right)=\frac{1}{\Gamma(s)} \int_{0}^{\infty} \frac{t^{s-1}}{e^{t}-1} \cdot L i_{k_{1}, k_{2}, \ldots, k_{n-1}}\left(e^{-t}\right) d t .
$$

(ii) For $\operatorname{Re}(s)>0$ and integers $n \geq 2, j \geq 0$, we have

$$
\int_{0}^{\infty} t^{s+j-1} L i_{k_{1}, k_{2}, \ldots, k_{n-1}}\left(e^{-t}\right) d t=\Gamma(s+j) \zeta\left(k_{1}, \ldots, k_{n-2} ; s+j+k_{n-1}\right) .
$$

Proof. To obtain (i), write the sum in the definition of $\zeta\left(k_{1}, k_{2}, \ldots\right.$, $\left.k_{n-1} ; s\right)$ as

$$
\sum_{0<m_{1}<\cdots<m_{n-1}} \frac{1}{m_{1}^{k_{1}} m_{2}^{k_{2}} \cdots m_{n-1}^{k_{n-1}}} \sum_{m_{n}=m_{n-1}+1}^{\infty} \frac{1}{m_{n}^{s}}
$$

and use the standard expression $\frac{1}{n^{s}}=\frac{1}{\Gamma(s)} \int_{0}^{\infty} e^{-n t} t^{s-1} d t$ to convert the inner sum into the integral. As for (ii), we only need to replace $L i_{k_{1}, k_{2}, \ldots, k_{n-1}}\left(e^{-t}\right)$ by its defining series

$$
\sum_{0<m_{1}<m_{2}<\cdots<m_{n-1}} \frac{e^{-m_{n-1} t}}{m_{1}^{k_{1}} m_{2}^{k_{2}} \cdots m_{n-1}^{k_{n-1}}}
$$

and use the same formula $\int_{0}^{\infty} e^{-m_{n-1} t} t^{s+j-1} d t=\frac{\Gamma(s+j)}{m_{n-1}^{s+j}}$ as above.

Now we state the basic

TheOREM 3. (i) For $\operatorname{Re}(s)>1$, we have

$$
\begin{array}{r}
\zeta\left(k_{1}, k_{2}, \ldots, k_{n-1} ; s\right)=\frac{1}{\Gamma\left(k_{1}\right) \cdots \Gamma\left(k_{n-1}\right) \Gamma(s)} \int_{0}^{\infty} \cdots \int_{0}^{\infty} d x_{1} \cdots d x_{n-1} d x_{n} . \\
x_{1}^{k_{1}-1} \cdots x_{n-1}^{k_{n-1}-1} x_{n}^{s-1} \frac{1}{e^{x_{1}+\cdots+x_{n}}-1} \cdot \frac{1}{e^{x_{2}+\cdots+x_{n}}-1} \cdots \frac{1}{e^{x_{n}}-1} .
\end{array}
$$

(ii) The zeta function $\zeta\left(k_{1}, k_{2}, \ldots, k_{n-1} ; s\right)$ can be analytically continued to a meromorphic function of $s$ in the whole complex plane. 
Proof. Writing the sum in the definition of $\zeta\left(k_{1}, k_{2}, \ldots, k_{n-1} ; s\right)$ as $\sum_{m_{1}=1}^{\infty} \sum_{m_{2}=m_{1}+1}^{\infty} \ldots \sum_{m_{n}=m_{n-1}+1}^{\infty}$, we can prove (i) by the repetition of the same calculation as in the proof of Proposition 2 (i). We prove (ii) by induction on $n$. The case of $n=1$ is the analytic continuation of the Riemann zeta function. Let $N$ be an arbitrary non-negative integer. Write

$$
\frac{1}{e^{t}-1}=\sum_{j=0}^{N} \frac{B_{j}}{j !} t^{j-1}+R_{N}(t)
$$

with

$$
R_{N}(t)=\sum_{j=N+1}^{\infty} \frac{B_{j}}{j !} t^{j-1},
$$

where $B_{j}$ are the classical Bernoulli numbers defined by this expansion. By Proposition 2 (ii), we have

$$
\begin{aligned}
& \int_{0}^{\infty} t^{s-1} L i_{k_{1}, k_{2}, \ldots, k_{n-1}}\left(e^{-t}\right) \sum_{j=0}^{N} \frac{B_{j}}{j !} t^{j-1} d t \\
& =\sum_{j=0}^{N} \frac{B_{j}}{j !} \Gamma(s+j-1) \zeta\left(k_{1}, \ldots, k_{n-2} ; s+j+k_{n-1}-1\right),
\end{aligned}
$$

and thus

$$
\begin{aligned}
& \Gamma(s) \zeta\left(k_{1}, k_{2}, \ldots, k_{n-1} ; s\right)=\int_{0}^{\infty} t^{s-1} L i_{k_{1}, k_{2}, \ldots, k_{n-1}}\left(e^{-t}\right) R_{N}(t) d t \\
& +\sum_{j=0}^{N} \frac{B_{j}}{j !} \Gamma(s+j-1) \zeta\left(k_{1}, \ldots, k_{n-2} ; s+j+k_{n-1}-1\right) .
\end{aligned}
$$

The second term in the right-hand side of (2) can be analytically continued to a meromorphic function in the whole $s$-plane by the induction assumption. Write the integral in (2) as a sum of two integrals:

$$
\int_{0}^{1} t^{s-1} L i_{k_{1}, k_{2}, \ldots, k_{n-1}}\left(e^{-t}\right) R_{N}(t) d t+\int_{1}^{\infty} t^{s-1} L i_{k_{1}, k_{2}, \ldots, k_{n-1}}\left(e^{-t}\right) R_{N}(t) d t .
$$


The second integral converges absolutely for any $s \in \mathbf{C}$ and represents an entire function of $s$. Using the estimates

$$
L i_{k_{1}, k_{2}, \ldots, k_{n-1}}\left(e^{-t}\right) \leq\left\{\begin{array}{r}
L i_{k_{1}, k_{2}, \ldots, k_{n-1}}(1)=\zeta\left(k_{1}, k_{2}, \ldots, k_{n-1}\right) \\
\quad \text { for } k_{n-1}>1 \\
L \underbrace{i_{1,1, \ldots, 1}}_{n-1}\left(e^{-t}\right)=\frac{1}{(n-1) !}\left(-\log \left(1-e^{-t}\right)\right)^{n-1} \\
\quad \text { for } k_{n-1}=1,
\end{array}\right.
$$

and

$$
R_{N}(t)=O\left(|t|^{N}\right) \quad(|t|<1),
$$

we see that the first integral converges absolutely for $\operatorname{Re}(s+N)>0$ and hence holomorphic as a function of $s$ in the same region. Since we can choose $N$ arbitrary large, this shows via (2) that the function $\zeta\left(k_{1}, k_{2}, \ldots, k_{n-1} ; s\right)$ has a meromorphic continuation to the whole $s$-plane.

By means of the inductive relation (2), we can, in principle, detect the location and the order of poles of $\zeta\left(k_{1}, k_{2}, \ldots, k_{n-1} ; s\right)$, which are in general quite complicated to describe. Possible poles lie at the integers $\leq 1$. Here we only state results at $s=1$ as a proposition.

Proposition 4. (i) Let the index set $\left(k_{1}, k_{2}, \ldots, k_{n-1}\right)$ be of the form $(k_{1}, \ldots, k_{i}, \underbrace{1,1, \ldots, 1}_{m})$ with $m \geq 0, i \geq 1$, and $k_{i}>1$. Then, the order of pole of $\zeta\left(k_{1}, k_{2}, \ldots, k_{n-1} ; s\right)$ at $s=1$ is $m+1$ and the leading coefficient is $\zeta\left(k_{1}, k_{2}, \ldots, k_{i}\right)(i=n-m-1)$, i.e.

$$
\zeta\left(k_{1}, k_{2}, \ldots, k_{n-1} ; s\right)=\frac{\zeta\left(k_{1}, k_{2}, \ldots, k_{i}\right)}{(s-1)^{m+1}}+O\left((s-1)^{-m}\right) .
$$

(ii) For $\left(k_{1}, k_{2}, \ldots, k_{n-1}\right)=(1,1, \ldots, 1)$, we have

$$
\Gamma(s) \zeta(\underbrace{1,1, \ldots, 1}_{n-1} ; s)=\frac{1}{(s-1)^{n}}+O(s-1) .
$$

Proof. (i) The integral in (2) is holomorphic at $s=1$ even when $N=0$, in which case the relation (2) divided by $\Gamma(s)$ reads

$$
\begin{aligned}
\zeta\left(k_{1}, k_{2}, \ldots, k_{n-1} ; s\right)=\frac{1}{\Gamma(s)} & \int_{0}^{\infty} t^{s-1} L i_{k_{1}, k_{2}, \ldots, k_{n-1}}\left(e^{-t}\right) R_{0}(t) d t \\
& +\frac{1}{s-1} \zeta\left(k_{1}, \ldots, k_{n-2} ; s+k_{n-1}-1\right) .
\end{aligned}
$$


By our definition of $m$, the function $\zeta\left(k_{1}, \ldots, k_{n-m-2} ; s+k_{n-m-1}-1\right)$ is holomorphic at $s=1$ with the value $\zeta\left(k_{1}, k_{2}, \ldots, k_{n-m-1}\right)$. From this the case of $m=0$ follows, and we can easily obtain the proposition by induction on the number of indices $k_{i}$, starting with

$$
\zeta(s)=\frac{1}{s-1}+O(1) .
$$

(ii) By Proposition 2 (i) and Lemma 1 (ii), we have

$$
\Gamma(s) \zeta(\underbrace{1,1, \ldots, 1}_{n-1} ; s)=\frac{(-1)^{n-1}}{(n-1) !} \int_{0}^{\infty} \frac{t^{s-1}}{e^{t}-1}\left(\log \left(1-e^{-t}\right)\right)^{n-1} d t .
$$

Let $\zeta^{*}(\underbrace{1,1, \ldots, 1}_{n-1} ; s)$ be defined by

$$
\Gamma(s) \zeta^{*}(\underbrace{1,1, \ldots, 1}_{n-1} ; s)=\frac{(-1)^{n-1}}{(n-1) !} \int_{0}^{\infty} \frac{t^{s-1}}{e^{t}-1}\left(\log \left(\frac{1-e^{-t}}{t}\right)\right)^{n-1} d t .
$$

By the standard method already used to prove the analytic continuation of $\zeta\left(k_{1}, k_{2}, \ldots, k_{n-1} ; s\right)$, we see that the function $\zeta^{*}(\underbrace{1,1, \ldots, 1}_{n-1} ; s)$ is continued to an entire function in the whole s-plane. Now we claim that the following relation holds:

$$
\begin{aligned}
\Gamma(s) \zeta(\underbrace{1, \ldots, 1}_{n-1} ; s)= & \frac{(-1)^{n-1}}{(n-1) !} \cdot \frac{d^{n-1}}{d s^{n-1}}(\Gamma(s) \zeta(s)) \\
& +\sum_{j=0}^{n-2} \frac{(-1)^{j}}{j !} \cdot \frac{d^{j}}{d s^{j}}\left(\Gamma(s) \zeta^{*}(\underbrace{1, \ldots, 1}_{n-1-j} ; s)\right) .
\end{aligned}
$$

This can be seen by using

$$
\left(\log \left(1-e^{-t}\right)\right)^{n-1}=\sum_{j=0}^{n-1}\left(\begin{array}{c}
n-1 \\
j
\end{array}\right)(\log t)^{j}\left(\log \left(\frac{1-e^{-t}}{t}\right)\right)^{n-1-j}
$$

and

$$
\begin{aligned}
& \int_{0}^{\infty} \frac{t^{s-1}}{e^{t}-1}(\log t)^{j}\left(\log \left(\frac{1-e^{-t}}{t}\right)\right)^{n-1-j} d t \\
&=(-1)^{n-1-j}(n-1-j) ! \frac{d^{j}}{d s^{j}}\left\{\Gamma(s) \zeta^{*}(\underbrace{1, \ldots, 1}_{n-1-j} ; s)\right\} .
\end{aligned}
$$


Using (4) and the formula

$$
\Gamma(s) \zeta(s)=\frac{1}{s-1}+O(s-1)
$$

which is equivalent to the more familiar ("Kronecker limit formula for $\zeta(s)$ ", see e.g. Siegel $[\mathrm{S}]$ )

$$
\zeta(s)=\frac{1}{s-1}+\gamma+O(s-1) \quad(\gamma=\text { Euler's constant })
$$

we conclude that

$$
\Gamma(s) \zeta(\underbrace{1, \ldots, 1}_{n-1} ; s)=\frac{1}{(s-1)^{n}}+O(1) .
$$

Let $c_{0}^{(n)}$ be the constant term on the right-hand side. From the relation (obtained from (2) by putting $N=0$ and $\forall k_{i}=1$ )

$$
\begin{aligned}
& \zeta(\underbrace{1, \ldots, 1}_{n-1} ; s) \\
= & \frac{1}{\Gamma(s)} \int_{0}^{\infty} t^{s-1} L \underbrace{i_{1,1, \ldots, 1}}_{n-1}\left(e^{-t}\right) R_{0}(t) d t+\frac{1}{s-1} \zeta(\underbrace{1, \ldots, 1}_{n-2} ; s),
\end{aligned}
$$

we obtain

$$
\frac{1}{(s-1)^{n}}+c_{0}^{(n)}+O(s-1)=\frac{1}{(s-1)^{n}}+\frac{c_{0}^{(n-1)}}{s-1}+O(1),
$$

hence we necessarily have $c_{0}^{(n-1)}=0$. Since $n$ is arbitrary, this completes the proof of the Proposition.

Remarks. 1. The values of the holomorphic function $\zeta^{*}(\underbrace{1,1, \ldots, 1}_{r}, s)$ at non-positive integers are evaluated in terms of Bernoulli numbers:

$$
\zeta^{*}(\underbrace{1,1, \ldots, 1}_{r}, 1-m)=\frac{(-1)^{r+m-1}}{r !} \cdot \frac{D_{m}^{(r)}}{m} \quad(m \geq 1),
$$

where $D_{m}^{(r)}$ is defined by

$$
\frac{1}{e^{t}-1} \cdot\left\{\log \left(\frac{1-e^{-t}}{t}\right)\right\}^{r}=\sum_{m=1}^{\infty} D_{m}^{(r)} \cdot \frac{t^{m-1}}{m !}
$$


(note that $D_{m}^{(r)}=0$ for $1 \leq m \leq r-1$ ). It is easy to see that (noting $\left.\left(\log \left(\frac{1-e^{-t}}{t}\right)\right)^{\prime}=\frac{1}{e^{t}-1}-\frac{1}{t}\right)$

$$
D_{m}^{(1)}=\sum_{j=0}^{m-1}\left(\begin{array}{c}
m \\
j
\end{array}\right) \cdot B_{j} \cdot \frac{B_{m-j}}{m-j}
$$

and that

$$
D_{m}^{(r+1)}=\sum_{l=r}^{m-1}\left(\begin{array}{c}
m \\
l
\end{array}\right) \cdot D_{l}^{(r)} \cdot \frac{B_{m-l}}{m-l}
$$

for $r \geq 1$ and $m \geq r+1$. Hence $D_{m}^{(r)}$ is the sum of $(r+1)$-fold products of Bernoulli numbers.

2. As mentioned in the proof, Proposition 4 (ii) above can be regarded as a generalization of the "Kronecker limit formula for $\zeta(s)$ ". Moreover, we can obtain from the assertion (3) and the relation (5) the following formula giving the coefficients of the expansion at $s=1$ of $\Gamma(s) \zeta(s)$ :

Corollary 5. Let $c_{n}$ be defined by

$$
\Gamma(s) \zeta(s)=\frac{1}{s-1}+\sum_{n=1}^{\infty} c_{n}(s-1)^{n} .
$$

Then

$$
c_{n}=\frac{1}{n !} \int_{0}^{\infty}\left\{(\log t)^{n}-\left(\log \frac{t}{1-e^{-t}}\right)^{n}\right\}\left(\frac{1}{e^{t}-1}-\frac{1}{t}\right) d t .
$$

Proof. Near $s=1$, we can write

$$
\Gamma(s) \zeta(\underbrace{1, \ldots, 1}_{n} ; s)=\frac{1}{(s-1)^{n+1}}+\sum_{m=1}^{\infty} a_{m}^{(n)}(s-1)^{m}
$$

(we put $a_{0}^{(n)}=0$ for later use) and

$$
\int_{0}^{\infty} t^{s-1} L \underbrace{i_{1,1, \ldots, 1}}_{n}\left(e^{-t}\right) R_{0}(t) d t=\sum_{m=0}^{\infty} b_{m}^{(n)}(s-1)^{m},
$$

where

$$
b_{m}^{(n)}=\frac{1}{n ! m !} \int_{0}^{\infty}(\log t)^{m}\left(-\log \left(1-e^{-t}\right)\right)^{n}\left(\frac{1}{e^{t}-1}-\frac{1}{t}\right) d t
$$


(recall that $L i_{n}^{1,1, \ldots, 1}\left(e^{-t}\right)=\frac{1}{n !}\left(-\log \left(1-e^{-t}\right)\right)^{n}$ and $\left.R_{0}(t)=\frac{1}{e^{t}-1}-\frac{1}{t}\right)$. By

(5) (multiplied by $\Gamma(s)$, and $n$ replaced by $n+1$ ),

$$
\frac{1}{(s-1)^{n+1}}+\sum_{m=1}^{\infty} a_{m}^{(n)}(s-1)^{m}=\frac{1}{(s-1)^{n+1}}+\sum_{m=0}^{\infty}\left(b_{m}^{(n)}+a_{m+1}^{(n-1)}\right)(s-1)^{m} .
$$

Comparing the coefficients of $(s-1)^{m}$, we obtain

$$
a_{m}^{(n)}=b_{m}^{(n)}+a_{m+1}^{(n-1)} \quad \text { for } m \geq 0 .
$$

From this we have

$$
a_{m}^{(n)}=\sum_{i=0}^{n-1} b_{m+i}^{(n-i)}+a_{m+n}^{(0)}
$$

In particular, putting $m=0$ and using $a_{0}^{(n)}=0$, we finally have

$$
\begin{aligned}
c_{n}=a_{n}^{(0)} & =-\sum_{i=0}^{n-1} b_{i}^{(n-i)} \\
& =-\sum_{i=0}^{n-1} \frac{1}{(n-i) ! i !} \int_{0}^{\infty}(\log t)^{i}\left(-\log \left(1-e^{-t}\right)\right)^{n-i}\left(\frac{1}{e^{t}-1}-\frac{1}{t}\right) d t \\
& =-\frac{1}{n !} \int_{0}^{\infty}\left(\left(\log t-\log \left(1-e^{-t}\right)\right)^{n}-(\log t)^{n}\right)\left(\frac{1}{e^{t}-1}-\frac{1}{t}\right) d t \\
& =\frac{1}{n !} \int_{0}^{\infty}\left((\log t)^{n}-\left(\log \frac{t}{1-e^{-t}}\right)^{n}\right)\left(\frac{1}{e^{t}-1}-\frac{1}{t}\right) d t
\end{aligned}
$$

Conversely, one can prove (3) starting from this formula by tracing backwards the above argument.

\section{$\S 3$. Poly-Bernoulli numbers and zeta functions}

In $[\mathrm{Ka}]$, we introduced and studied "poly-Bernoulli numbers" which generalize the classical Bernoulli numbers. Poly-Bernoulli numbers $B_{n}^{(k)}$, with $k \in \mathbf{Z}$ and $n \in \mathbf{N}$, are by definition given by the following generating series:

$$
\frac{\operatorname{Li}_{k}\left(1-e^{-x}\right)}{1-e^{-x}}=\sum_{n=0}^{\infty} B_{n}^{(k)} \frac{x^{n}}{n !}
$$


where, for any integer $k, L i_{k}(z)$ denotes the formal power series (for the $k$-th polylogarithm if $k \geq 1$ and a rational function if $k \leq 0) \sum_{m=1}^{\infty} z^{m} / m^{k}$. When $k=1, B_{n}^{(1)}$ is the usual Bernoulli number $\left(B_{1}^{(1)}=\frac{1}{2}\right)$, and when $k \geq 1$, the left-hand side of (6) can be written in the form of "iterated integrals":

$$
\begin{aligned}
& e^{x} \cdot \underbrace{\frac{1}{e^{x}-1} \int_{0}^{x} \frac{1}{e^{x}-1} \int_{0}^{x} \cdots \frac{1}{e^{x}-1} \int_{0}^{x}}_{(k-1)-\text { times }} \frac{x}{e^{x}-1} d x d x \cdots d x \\
= & \sum_{n=0}^{\infty} B_{n}^{(k)} \frac{x^{n}}{n !} .
\end{aligned}
$$

In addition, we define the numbers $C_{n}^{(k)}$ by

$$
\frac{L i_{k}\left(1-e^{-x}\right)}{e^{x}-1}=\sum_{n=0}^{\infty} C_{n}^{(k)} \frac{x^{n}}{n !} .
$$

Since the left hand sides of (6) and (8) differs by a factor $e^{x}$, the numbers $B_{n}^{(k)}$ and $C_{n}^{(k)}$ are related by

$$
B_{n}^{(k)}=\sum_{m=0}^{n}\left(\begin{array}{c}
n \\
m
\end{array}\right) C_{m}^{(k)}, \quad C_{n}^{(k)}=\sum_{m=0}^{n}(-1)^{n-m}\left(\begin{array}{c}
n \\
m
\end{array}\right) B_{m}^{(k)} .
$$

Also, using

$$
\begin{aligned}
\frac{L i_{k}\left(1-e^{-x}\right)}{1-e^{-x}} & =e^{x} \frac{L i_{k}\left(1-e^{-x}\right)}{e^{x}-1}=\frac{L i_{k}\left(1-e^{-x}\right)}{e^{x}-1}+L i_{k}\left(1-e^{-x}\right) \\
& =\frac{L i_{k}\left(1-e^{-x}\right)}{e^{x}-1}+\int_{0}^{x} \frac{L i_{k-1}\left(1-e^{-x}\right)}{e^{x}-1} d x
\end{aligned}
$$

we have

$$
B_{n}^{(k)}=C_{n}^{(k)}+C_{n-1}^{(k-1)} .
$$

In paricular, specializing $k=2$ and using the fact that $C_{n}^{(1)}=0$ for odd $n \geq 3$, we have

$$
B_{n}^{(2)}=C_{n}^{(2)} \quad \text { for even } n \geq 4 .
$$


Now, we define the function $\xi_{k}(s)$ for $k \geq 1$ by

$$
\xi_{k}(s):=\frac{1}{\Gamma(s)} \int_{0}^{\infty} \frac{t^{s-1}}{e^{t}-1} L i_{k}\left(1-e^{-t}\right) d t
$$

The integral converges for $\operatorname{Re}(s)>0$. When $k=1, \xi_{1}(s)$ is equal to $s \zeta(s+1)$.

THEOREM 6. (i) The function $\xi_{k}(s)$ continues to an entire function of $s$, and the special values at non-positive integers are given by

$$
\xi_{k}(-m)=(-1)^{m} C_{m}^{(k)}=\sum_{\ell=0}^{m}(-1)^{\ell}\left(\begin{array}{c}
m \\
\ell
\end{array}\right) B_{\ell}^{(k)} \quad(m=0,1,2, \ldots) .
$$

(ii) The function $\xi_{k}(s)$ can be written in terms of the zeta functions $\zeta\left(k_{1}, k_{2}, \ldots, k_{n-1} ; s\right)$ as

$$
\begin{aligned}
\xi_{k}(s)=( & -1)^{k-1}\{\zeta(\underbrace{2,1, \ldots, 1}_{k-1} ; s)+\zeta(\underbrace{1,2,1, \ldots, 1}_{k-1} ; s)+\cdots+\zeta(\underbrace{1, \ldots, 1,2}_{k-1} ; s) \\
& +s \cdot \zeta(\underbrace{1,1, \ldots, 1}_{k-1} ; s+1)\}+\sum_{j=0}^{k-2}(-1)^{j} \zeta(k-j) \cdot \zeta(\underbrace{1,1, \ldots, 1}_{j} ; s) .
\end{aligned}
$$

Proof. (i) Write the integral in (10) as the sum of two integrals:

$$
\xi_{k}(s)=\frac{1}{\Gamma(s)} \int_{0}^{1} \frac{t^{s-1}}{e^{t}-1} L i_{k}\left(1-e^{-t}\right) d t+\frac{1}{\Gamma(s)} \int_{1}^{\infty} \frac{t^{s-1}}{e^{t}-1} L i_{k}\left(1-e^{-t}\right) d t .
$$

The second integral converges absolutely for any $s \in \mathbf{C}$ and hence the second term on the right-hand side vanishes at non-positive integers because $\Gamma(s)^{-1}$ so does. Then substituting the Taylor expansion (8) of $L i_{k}\left(1-e^{-t}\right) /\left(e^{t}-1\right)$ into the first integral, we obtain, by a standard method (cf. [Z3, §4 of Part 1]), the analytic continuation to an entire function and the desired expression for the special value $\xi_{k}(-m)$.

(ii) Consider the integral

$$
J(s)=\int_{0}^{\infty} \cdots \int_{0}^{\infty} x_{k}^{s-1} \cdot \frac{x_{1}+\cdots+x_{k}}{e^{x_{1}+\cdots+x_{k}}-1} \cdot \frac{1}{e^{x_{2}+\cdots+x_{k}}-1} \cdots \frac{1}{e^{x_{k}}-1} d x_{1} \cdots d x_{k} .
$$


By Theorem 3, we have

$$
\begin{aligned}
\text { (11) } J(s)= & \Gamma(s)\{\zeta(\underbrace{2,1, \ldots, 1}_{k-1} ; s)+\zeta(\underbrace{1,2, \ldots, 1}_{k-1} ; s)+\cdots \zeta(\underbrace{1, \ldots, 1,2}_{k-1} ; s)\} \\
& +\Gamma(s+1) \zeta(\underbrace{1,1, \ldots, 1}_{k-1} ; s+1) .
\end{aligned}
$$

On the other hand, integrating directly we can show

$$
\sum_{j=0}^{k-2}(-1)^{k-j} \zeta(k-j) \Gamma(s) \zeta(\underbrace{1,1, \ldots, 1}_{j} ; s) .
$$

In fact, since

$$
\frac{\partial}{\partial x_{1}} L i_{2}\left(1-e^{-x_{1}-\cdots-x_{k}}\right)=\frac{x_{1}+\cdots+x_{k}}{e^{x_{1}+\cdots+x_{k}}-1}
$$

we have

$$
\begin{aligned}
J(s)= & \int_{0}^{\infty} \cdots \int_{0}^{\infty} x_{k}^{s-1} \cdot\left\{L i_{2}(1)-L i_{2}\left(1-e^{-x_{2}-\cdots-x_{k}}\right)\right\} . \\
& \frac{1}{e^{x_{2}+\cdots+x_{k}-1} \cdots \frac{1}{e^{x_{k}}-1} d x_{2} \cdots d x_{k}} \\
= & \zeta(2) \Gamma(s) \zeta(\underbrace{1, \ldots, 1}_{k-2} ; s)-\int_{0}^{\infty} \cdots \int_{0}^{\infty} x_{k}^{s-1} \cdot \frac{\partial}{\partial x_{2}} L i_{3}\left(1-e^{-x_{2}-\cdots-x_{k}}\right) . \\
& \frac{1}{e^{x_{3}+\cdots+x_{k}-1}} \cdots \frac{1}{e^{x_{k}-1}} d x_{2} \cdots d x_{k} .
\end{aligned}
$$

Continuing in the same manner, we obtain (12). Hence, together with (11), we have proved (ii).

Combining the above theorem (i) and the formula (9) preceding the theorem, we obtain

COROLlary 7. The poly-Bernoulli number $B_{n}^{(k)}$ is expressed as

$$
B_{n}^{(k)}=(-1)^{n}\left(\xi_{k}(-n)-\xi_{k-1}(-n+1)\right) \quad \text { for } n \geq 1 .
$$

In particular,

$$
B_{n}^{(2)}=\xi_{2}(-n) \quad \text { for even } n \geq 4
$$


Remarks. (i) Using the integral expressions (10) and Proposition 2 (i), we can derive from the above theorem (ii) a kind of functional equation relating polylogarithm $L i_{k}(1-z)$ to multilogarithms:

$$
\begin{aligned}
& L i_{k}(1-z) \\
& =(-1)^{k-1}\{L \underbrace{i_{2,1, \ldots, 1}}_{k-1}(z)+L \underbrace{i_{1,2,1, \ldots, 1}}_{k-1}(z)+\cdots+L \underbrace{i_{1, \ldots, 1,2}}_{k-1}(z)\} \\
& +\sum_{j=0}^{k-2} \frac{\zeta(k-j)}{j !}(\log (1-z))^{j}-\frac{1}{(k-1) !} \log (z)(\log (1-z))^{k-1} \\
& \quad(0<z<1) .
\end{aligned}
$$

When $k=2$, this is nothing but the well-known functional equation for the di-logarithm function (see e.g. Levin [L] p.5 (1.11)):

$$
L i_{2}(z)+L i_{2}(1-z)=\zeta(2)-\log z \log (1-z)
$$

Conversely, starting from this functional equation for $L i_{2}$, we can easily derive by induction the above equation for $L i_{k}$, and thus obtain another proof of Theorem 6 (ii).

(ii) As a further generalization of $B_{n}^{(k)}$, we may define the number $B_{n}^{\left(k_{1}, k_{2}, \ldots, k_{m}\right)}$ by

$$
\frac{L i_{k_{1}, k_{2}, \ldots k_{m}}\left(1-e^{-x}\right)}{\left(1-e^{-x}\right)^{m}}=\sum_{n=0}^{\infty} B_{n}^{\left(k_{1}, k_{2}, \ldots, k_{m}\right)} \frac{x^{n}}{n !} .
$$

Note that this also generalizes the "higher order Bernoulli numbers" ( $c f$. e.g. [C]) because by Lemma 1 (ii), the case of $k_{1}=k_{2}=\cdots=k_{m}=1$ becomes

$$
\sum_{n=0}^{\infty} B_{n}^{(\overbrace{1,1, \ldots, 1}^{m}} \frac{x^{n}}{n !}=\frac{1}{m !}\left(\frac{-x}{e^{-x}-1}\right)^{m} .
$$

(We inadvertently committed an offence in [Ka] of using the same notation $B_{n}^{(k)}$ as in [C] for another series of numbers. Since we never discuss the higher order Bernoulli numbers in the present paper, we adopted the same notation in order not to cause further confusion.) 


\section{$\S 4$. Values at positive arguments and multiple zeta values}

In this section, we introduce a generalization of our zeta function (10) for multiple index set $\left(k_{1}, \ldots, k_{r}\right)$. We derive formulas for the values at positive arguments, as well as a series of relations among multiple zeta values.

Let $\left(k_{1}, \ldots, k_{r}\right)$ be any $r$-tuple of positive integers. Define

$$
\xi\left(k_{1}, k_{2}, \ldots, k_{r} ; s\right)=\frac{1}{\Gamma(s)} \int_{0}^{\infty} \frac{t^{s-1}}{e^{t}-1} \cdot L i_{k_{1}, k_{2}, \ldots, k_{r}}\left(1-e^{-t}\right) d t .
$$

This is absolutely convergent for $\operatorname{Re}(s)>0$. Note that $\xi(k ; s)=\xi_{k}(s)$ in our previous notation. First we generalize Theorem 6 (ii) to the function $\xi(\underbrace{1, \ldots, 1}_{r-1}, k ; s)$ for any $r \geq 1$ and $k \geq 1$.

TheOREM 8. For $r, k \geq 1$, we have

$$
\begin{aligned}
& \xi(\underbrace{1, \ldots, 1}_{r-1}, k ; s) \\
=(-1)^{k-1} & \sum_{\substack{a_{1}+\ldots+a_{k}=r \\
\forall a_{j} \geq 0}}\left(\begin{array}{c}
s+a_{k}-1 \\
a_{k}
\end{array}\right) \zeta\left(a_{1}+1, \ldots, a_{k-1}+1 ; a_{k}+s\right) \\
& +\sum_{j=0}^{k-2}(-1)^{j} \zeta(\underbrace{1, \ldots, 1}_{r-1}, k-j) \zeta(\underbrace{1, \ldots, 1}_{j} ; s) .
\end{aligned}
$$

Proof. Consider the integral

$$
\begin{aligned}
J(s)=\int_{0}^{\infty} \cdots \int_{0}^{\infty} d x_{1} \cdots d x_{k} \cdot x_{k}^{s-1} . \\
\\
\qquad \frac{\left(x_{1}+\cdots+x_{k}\right)^{r}}{e^{x_{1}+\cdots+x_{k}}-1} \cdot \frac{1}{e^{x_{2}+\cdots+x_{k}}-1} \cdots \cdot \frac{1}{e^{x_{k}}-1} .
\end{aligned}
$$

Successive integration using the identities

$$
\left(x_{1}+\cdots+x_{k}\right)^{r}=r ! L \underbrace{1, \ldots, 1}_{r}\left(1-e^{-\left(x_{1}+\cdots+x_{k}\right)}\right)
$$

and

$$
\frac{1}{e^{x_{\nu}+\cdots+x_{k}}-1} L \underbrace{i_{1, \ldots, 1, \nu}}_{r}\left(1-e^{-\left(x_{\nu}+\cdots+x_{k}\right)}\right)
$$




$$
=\frac{\partial}{\partial x_{\nu}} L \underbrace{i_{1, \ldots, 1, \nu+1}^{1, \nu}}_{r}\left(1-e^{-\left(x_{\nu}+\cdots+x_{k}\right)}\right)
$$

leads, as in the proof of Theorem 6 , to the expression

$$
\begin{aligned}
J(s)= & r ! \Gamma(s)\{\zeta(\underbrace{1, \ldots, 1}_{r-1}, 2) \zeta(\underbrace{1, \ldots, 1}_{k-2} ; s)-\zeta(\underbrace{1, \ldots, 1}_{r-1}, 3) \zeta(\underbrace{1, \ldots, 1}_{k-3} ; s) \\
& \left.+\cdots+(-1)^{k} \zeta(\underbrace{1, \ldots, 1}_{r-1}, k) \zeta(s)+(-1)^{k+1} \xi(\underbrace{1, \ldots, 1}_{r-1}, k ; s)\right\} .
\end{aligned}
$$

On the other hand, expanding $\left(x_{1}+\cdots+x_{k}\right)^{r}$ into the sum of monomials and using Theorem 3 (i), we have

$$
J(s)=\sum_{\substack{a_{1}+\ldots+a_{k}=r \\ \forall a_{j} \geq 0}} \frac{r ! \Gamma\left(s+a_{k}\right)}{\Gamma\left(a_{k}+1\right)} \zeta\left(a_{1}+1, \ldots, a_{k-1}+1 ; a_{k}+s\right) .
$$

Comparing the two expressions of $J(s)$, we obtain the desired identity.

Now we state and prove the result on the special values at positive arguments.

TheOREM 9. (i) Let $m \geq 0, r \geq 1$, and $k \geq 1$ be integers. Then

$$
\begin{aligned}
& \xi(\underbrace{1, \ldots, 1}_{r-1}, k ; m+1) \\
= & \sum_{\substack{a_{1}+\cdots+a_{k}=m \\
\forall a_{j} \geq 0}}\left(\begin{array}{c}
a_{k}+r \\
r
\end{array}\right) \zeta\left(a_{1}+1, \ldots, a_{k-1}+1 ; a_{k}+r+1\right) .
\end{aligned}
$$

(ii) Let $m, r \geq 1, k \geq 2$ be integers. Then,

$$
\begin{aligned}
& \xi(\underbrace{1, \ldots, 1}_{r-1}, k ; m+1)+(-1)^{k} \xi(\underbrace{1, \ldots, 1}_{m-1}, k ; r+1) \\
= & \sum_{j=0}^{k-2}(-1)^{j} \zeta(\underbrace{1, \ldots, 1}_{r-1}, k-j) \zeta(\underbrace{1, \ldots, 1}_{m-1}, 2+j) .
\end{aligned}
$$


Proof. First we prove (ii). Using the identity $L \underbrace{i_{1, \ldots, 1}}_{m}\left(1-e^{-t}\right)=\frac{t^{m}}{m !}$, we have

$$
\begin{aligned}
& \xi(\underbrace{1, \ldots, 1}_{r-1}, k ; m+1) \\
& =\int_{0}^{\infty} L \underbrace{i_{1, \ldots, 1}, k}_{r-1}\left(1-e^{-t}\right) L \underbrace{i_{1, \ldots, 1}}_{m}\left(1-e^{-t}\right) \cdot \frac{1}{e^{t}-1} d t
\end{aligned}
$$

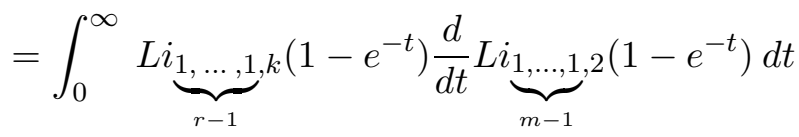

$$
\begin{aligned}
& =\zeta(\underbrace{1, \ldots, 1}_{r-1}, k) \zeta(\underbrace{1, \ldots, 1}_{m-1}, 2) \\
& -\int_{0}^{\infty} \frac{d}{d t} L \underbrace{i_{1, \ldots, 1}, k}_{r-1}\left(1-e^{-t}\right) L \underbrace{i_{1, \ldots, 1,2}}_{m-1}\left(1-e^{-t}\right) d t \\
& =\zeta(\underbrace{1, \ldots, 1}_{r-1}, k) \zeta(\underbrace{1, \ldots, 1}_{m-1}, 2) \\
& -\int_{0}^{\infty} L \underbrace{i_{1, \ldots, 1}, k-1}_{r-1}\left(1-e^{-t}\right) \frac{d}{d t} L \underbrace{i_{1, \ldots, 1}, 3}_{m-1}\left(1-e^{-t}\right) d t \\
& =\zeta(\underbrace{1, \ldots, 1}_{r-1}, k) \zeta(\underbrace{1, \ldots, 1}_{m-1}, 2)-\zeta(\underbrace{1, \ldots, 1}_{r-1}, k-1) \zeta(\underbrace{1, \ldots, 1}_{m-1}, 3) \\
& +\int_{0}^{\infty} L \underbrace{i_{1, \ldots, 1}, k-2}_{r-1}\left(1-e^{-t}\right) \frac{d}{d t} L \underbrace{i_{1, \ldots, 1,4}}_{m-1}\left(1-e^{-t}\right) d t \\
& =\cdots \\
& =\sum_{j=0}^{k-2}(-1)^{j} \zeta(\underbrace{1, \ldots, 1}_{r-1}, k-j) \zeta(\underbrace{1, \ldots, 1}_{m-1}, 2+j) \\
& +(-1)^{k+1} \int_{0}^{\infty} L \underbrace{i_{1}^{1, \ldots, 1}}_{r}\left(1-e^{-t}\right) L \underbrace{i_{1, \ldots, 1}, k}_{m-1}\left(1-e^{-t}\right) \frac{d t}{e^{t}-1} \\
& =\sum_{j=0}^{k-2}(-1)^{j} \zeta(\underbrace{1, \ldots, 1}_{r-1}, k-j) \zeta(\underbrace{1, \ldots, 1}_{m-1}, 2+j) \\
& -(-1)^{k} \xi(\underbrace{1, \ldots, 1}_{m-1}, k ; r+1),
\end{aligned}
$$


thus obtain the identity (14). To deduce (i), put $s=m+1$ in Theorem 8 . Then we have

$$
\begin{aligned}
& \xi(\underbrace{1, \ldots, 1}_{r-1}, k ; m+1) \\
&=(-1)^{k-1} \sum_{\substack{a_{1}+\ldots+a_{k}=r \\
\forall a_{j} \geq 0}}\left(\begin{array}{c}
a_{k}+m \\
m
\end{array}\right) \zeta\left(a_{1}+1, \ldots, a_{k-1}+1 ; a_{k}+m+1\right) \\
&+\sum_{j=0}^{k-2}(-1)^{j} \zeta(\underbrace{1, \ldots, 1}_{r-1}, k-j) \zeta(\underbrace{1, \ldots, 1}_{j} ; m+1) .
\end{aligned}
$$

Comparing this with the identity (14) just proved, and using the duality $(c f .[\mathrm{Z} 2])$

$$
\zeta(\underbrace{1, \ldots, 1}_{j} ; m+1)=\zeta(\underbrace{1, \ldots, 1}_{m-1} ; j+2),
$$

we obtain the formula (13) for $m \geq 1$. When $m=0$, (13) is a direct consequence from the definition and the above mentioned duality.

Putting $r=1$ in (13) and $m=r=1$ in (14), we have respectively

Corollary 10. (i) ${ }^{1}$ For $k \geq 1$ and $m \geq 0$,

$$
\xi_{k}(m+1)=\sum_{\substack{a_{1}+\ldots+a_{k}=m \\ \forall a_{j} \geq 0}}\left(a_{k}+1\right) \zeta\left(a_{1}+1, \ldots, a_{k-1}+1 ; a_{k}+2\right)
$$

(ii) If $k$ is even and $k \geq 2$, then

$$
\xi_{k}(2)=\frac{1}{2} \sum_{i=0}^{k-2}(-1)^{i} \zeta(i+2) \zeta(k-i)
$$

We note here that $\xi_{k}(1)=\zeta(k+1)$ by the definition (10). Combination of the two formulas in Theorem 9 yields many relations among multiple zeta values:

\footnotetext{
${ }^{1}$ Recently, Y. Ohno [O] obtained an another expression of $\xi_{k}(m+1)$ as a by-product of his simultaneous generalization of "duality" and "sum formula" for multiple zeta values.
} 
Corollary 11. For integers $m, r \geq 1$ and $k \geq 2$, we have

$$
\begin{aligned}
& \sum_{\substack{a_{1}+\ldots+a_{k}=m \\
\forall a_{j} \geq 0}}\left(\begin{array}{c}
a_{k}+r \\
r
\end{array}\right) \zeta\left(a_{1}+1, \ldots, a_{k-1}+1 ; a_{k}+r+1\right) \\
&+(-1)^{k} \sum_{\substack{a_{1}+\ldots+a_{k}=r \\
\forall a_{j} \geq 0}}\left(\begin{array}{c}
a_{k}+m \\
m
\end{array}\right) \zeta\left(a_{1}+1, \ldots, a_{k-1}+1 ; a_{k}+m+1\right) \\
&=\sum_{j=0}^{k-2}(-1)^{j} \zeta(\underbrace{1, \ldots, 1}_{r-1}, k-j) \zeta(\underbrace{1, \ldots, 1}_{m-1}, 2+j) .
\end{aligned}
$$
$(1.8))$

In particular, the case of $k=2$ yields (the formula appeared in [HWN]

$$
\begin{aligned}
& \sum_{i=0}^{m}\left(\begin{array}{c}
r+i \\
i
\end{array}\right) \zeta(m+1-i, r+1+i) \\
& \quad+\sum_{j=0}^{r}\left(\begin{array}{c}
m+j \\
j
\end{array}\right) \zeta(r+1-j, m+1+j) \\
& =\zeta(m+1) \zeta(r+1),
\end{aligned}
$$

and further specialization $m=r$ gives

$$
\sum_{i=0}^{m}\left(\begin{array}{c}
m+i \\
i
\end{array}\right) \zeta(m+1-i, m+1+i)=\frac{1}{2} \zeta(m+1)^{2} .
$$

\section{$\S 5$. Some problems}

In conclusion, we discuss some further problems.

(i) For a general index set $\left(k_{1}, \ldots, k_{r}\right)$, is the function $\xi\left(k_{1}, \ldots, k_{r} ; s\right)$ also expressed by multiple zeta functions as in Theorem 8? One may deduce as a consequence further relations among multiple zeta values.

(ii) The above problem (i) will be affirmatively answered if one can give a suitable functional equation for the multi-logarithm functions $L i_{k_{1}, k_{2}, \ldots, k_{r}}(z)$ under the substitution $z \mapsto 1-z$. What can one expect about functional equations of $L i_{k_{1}, k_{2}, \ldots, k_{r}}(z)$ ? We illustrate one example of the functional equation satisfied by the multi-logarithm function and its consequence. Namely, we have

$$
L i_{2,1}(1-z)=2 L i_{3}(z)-\log z \cdot L i_{2}(z)-\zeta(2) \log z-2 \zeta(3) .
$$


Differentiating both sides, this reduces to the functional equation between $L i_{2}(z)$ and $L i_{2}(1-z)$ which was recalled at the end of $\S 3$. From this identity, we obtain

$$
\xi(2,1 ; s)=2 \zeta(3 ; s)+s \zeta(2 ; s+1)+\zeta(2) s \zeta(s+1)-2 \zeta(3) \zeta(s) .
$$

(iii) May the values at positive integers $\xi\left(k_{1}, \ldots, k_{r} ; m\right)$ enjoy a certain kind of "duality" like the one in Theorem 9 (ii)?

(iv) Determine the principal part of the arbitrary multi-zeta functions $\zeta\left(k_{1}, \ldots, k_{n-1} ; s\right)$ at the pole $s=1$ (or more generally at each possible pole). This would lead, together with Theorem 8 , to certain non-trivial relations, perhaps including the "sum conjecture" ([Z]), among the multiple zeta values.

\section{REFERENCES}

[A] Arnold, V. I., The Vassiliev theory of discriminants and knots, in ECM volume, Progress in Math., 120 (1994), 3-29.

[BBB] Borwein, J. M., Bradley, D. M. and Broadhurst, D. J., Evaluations of $k$-fold Euler/Zagier sums: a compendium of results for arbitrary $k$, hep-th/9611004.

[BBG] Borwein, D., Borwein, J. M. and Girgensohn, R., Explicit evaluation of Euler sums, Proc. Edin. Math. Soc., 38 (1995), 277-294.

[Br] Broadhurst, D. J., Conjectured enumeration of irreducible multiple zeta values, from knots and Feynman diagrams, hep-th/9612012.

[C] Carlitz, L., Some theorems on Bernoulli numbers of higher order, Pacific J. Math., 2 (1952), 127-139.

[H] Hoffman, M., Multiple harmonic series, Pacific J. Math., 152 (1992), 275-290.

[HWN] Huard, J. G., Williams, K. S. and Nan-Yue, Z., On Tornheim's double series, Acta Arithmetica, 65-2 (1996), 105-117.

[J] Jordan, Ch., Calculus of Finite Differences, Chelsea Publ. Co., New York, 1950.

[Ka] Kaneko, M., Poly-Bernoulli numbers, J. Th. Nombre Bordeaux, 9 (1997), 199-206.

$[\mathrm{LM}]$ Le, T. Q. T. and Murakami, J., Kontsevich's integral for the Homfly polynomial and relations between values of multiple zeta functions, Topology and its Applications, 62 (1995), 193-206.

[L] Lewin, L., Polylogarithms and Associated Functions, Tata Institute, Bombay, 1980.

[O] Ohno, Y., A generalization of the duality and the sum formula on the multiple zeta values, J. Number Th., 74 (1999), 39-43.

[S] Siegel, C. L., Advanced Analytic Number Theory, Chelsea Publ. Co., New York, 1950.

[Z] Zagier, D., Multiple zeta values, in preparation. 
[Z2] Zagier, D., Values of zeta functions and their applications, in ECM volume, Progress in Math., 120 (1994), 497-512.

[Z3] Zagier, D., Zetafunktionen und quadratische Körper, Springer, 1981.

Tsuneo Arakawa

Department of Mathematics

Rikkyo University

Ikebukuro, Tokyo 171-8501

Japan

tsuneo@rkmath.rikkyo.ac.jp

Masanobu Kaneko

Graduate School of Mathematics

Kyushu University

Fukuoka, 812-8581

Japan

mkaneko@math.kyushu-u.ac.jp 